Article

\title{
A Comparison of Methods for Determining the Time Step When Propagating with the Lanczos Algorithm
}

\author{
N. Mohankumar ${ }^{1}$ and Tucker Carrington ${ }^{2, *}$ \\ 1 40, DJ Nagar, Peelamedu 6410004, India; kovainmk@gmail.com \\ 2 Chemistry Department, Queen's University, Kingston, ON K7L 3N6, Canada \\ * Correspondence: tucker.carrington@queensu.ca
}

Received: 15 October 2019; Accepted: 11 November 2019; Published: 15 November 2019

\begin{abstract}
To use the short iterative Lanczos algorithm to solve the time-dependent Schroedinger equation, one must choose, for a given Lanczos space size, a time step. We compare the derivation of the well-known Lubich and Hochbruck time step from SIAM J. Numer. Anal. 34 (1997) 1911 with the a priori time step we proposed in Mohankumar and Carrington (MC) Comput. Phys. Commun., 181 (2010) 1859 and demonstrate that the MC time step is somewhat larger, i.e., that the MC error bound is tighter. In addition, we use the MC approach to derive an error bound and time step for imaginary time propagation. The error bound we derive is much tighter than the error bound of Stewart and Leyk.
\end{abstract}

Keywords: short iterative Lanczos algorithm; propagation; Chebyshev error bounds

\section{Introduction}

The short iterative Lanczos (SIL) algorithm [1] for solving the time-dependent Schroedinger equation (TDSE) (in atomic units),

$$
i \frac{\partial}{\partial t} \psi(x, t)=\hat{H} \psi(x, t),
$$

is widely used in chemical physics and other fields. To solve the TDSE, the first step is to represent $\hat{H}$ and $\psi(x, t)$ in a (here orthonormal) basis, to obtain,

$$
i \frac{\partial \mathbf{a}(t)}{\partial t}=\mathbf{H a}(t)
$$

where

$$
\psi(x, t)=\sum_{k} a_{k}(t) \phi_{k}(x),
$$

and

$$
H_{k^{\prime}, k}=\left\langle\phi_{k^{\prime}}|H| \phi_{k}\right\rangle .
$$

solving the TDSE enables one to compute photodissociation cross section, rate constants, etc. [2-7]. To use SIL, Equation (2) is solved by writing $\mathbf{a}(t)=\mathbf{Q}_{\mathbf{m}} \mathbf{c}(t)$, and computing

$$
\mathbf{c}(t)=\mathbf{Q}_{\mathbf{m}}^{\mathbf{T}} \mathbf{a}(t) \approx e^{-i t \mathbf{T}_{\mathbf{m}}} \mathbf{c}(\mathbf{0}),
$$


where $\mathbf{Q}_{\mathbf{m}}$ is the matrix of $m$ Lanczos vectors,

$$
\mathrm{HQ}_{\mathrm{m}}=\mathbf{Q}_{\mathrm{m}+\mathbf{1}} \hat{\mathrm{T}}_{\mathrm{m}}
$$

and $\hat{\mathbf{T}}_{m}$ is a tridiagonal matrix,

$$
\hat{\mathbf{T}}_{m}=\left[\begin{array}{ccccc}
\alpha_{1} & \beta_{1} & 0 & \cdots & 0 \\
\beta_{1} & \alpha_{2} & \beta_{2} & \cdots & 0 \\
0 & \beta_{2} & \alpha_{3} & \beta_{3} & \cdots \\
\vdots & \vdots & \vdots & \vdots & \vdots \\
0 & \cdots & \beta_{m-2} & \alpha_{m-1} & \beta_{m-1} \\
0 & \cdots & 0 & \beta_{m-1} & \alpha_{m} \\
0 & \cdots & 0 & 0 & \beta_{m}
\end{array}\right]
$$

Note that

$$
\mathbf{T}_{\mathrm{m}}=\mathbf{Q}_{\mathrm{m}}^{\mathrm{T}} \mathbf{H Q}_{\mathrm{m}} .
$$

The notation used here is the same as the notation of [8]. $m$ is small enough that loss of orthogonality of the Lanczos vectors is unimportant. In this paper, we shall assume that $\mathbf{a}(t=0)$ is the Lanczos starting vector. In that case

$$
\mathbf{c}(0)=\mathbf{c}_{0}=\left[\begin{array}{c}
1 \\
0 \\
\vdots \\
0
\end{array}\right] \text {. }
$$

To use the SIL algorithm, one chooses a value of $m$, propagates from $t=0$ to $t=\Delta t$, and then uses $\mathbf{a}(\Delta t)$ as the starting vector and propagates again from $t=\Delta t$ to $t=2 \Delta t$, etc. For a fixed $m$, small enough that loss of orthogonality is not a problem, one must choose $\Delta t$. It is advantageous to make $\Delta t$ as large as possible, but $\Delta t$ must be chosen so that the size of the error at each step is acceptably small. Mathematicians tend to be familiar with the approach of Lubich (L) and Hochbruck [9-11] for choosing $\Delta t$.

In 2010, we (Mohankumar and Carrington-MC) derived a slightly different equation for choosing $\Delta t$ [8]. In this paper, we compare the derivations of the Lubich and MC equations and show that $\Delta t^{M C}$ is somewhat larger than $\Delta t^{L}$ and therefore that using $\Delta t^{M C}$ reduces the cost of propagating. In addition, we derive and test a new equation for $\Delta t$ for propagating in imaginary time.

\section{Comparing the Derivations}

\subsection{Common Starting Point}

Although the $\mathrm{L}$ and $\mathrm{MC}$ equations for $\Delta t$ are very similar, their derivations are different. Both derivations begin with a link between an error bound for a Lanczos approximation to a function of a matrix applied to a vector and an error for an approximation of the same function by a polynomial of a certain degree. In [8], we began with a result of Stewart and Leyk [12]. It is very similar to Theorem 2.9 of [9]. According to Theorem 2.9. for any complex-valued function $f$ defined on an interval $[a, b]$ that contains the eigenvalues of the Hermitian matrix $\mathbf{A}$, the error of the Lanczos approximation to $f(\mathbf{A}) \mathbf{v}$ is bounded by

$$
|| \mathbf{Q}_{\mathbf{m}} \mathbf{f}\left(\mathbf{T}_{\mathbf{m}}\right) \mathbf{e}_{\mathbf{1}}-f(\mathbf{A}) \mathbf{v}\left|\leq 2 \inf _{p_{m-1}} \max _{x \in[a, b]}\right| p_{m-1}(z)-f(z) \mid .
$$


Here, $\mathbf{v}$ is a unit vector and the infimum is taken over all polynomials of degree at most $(m-1)$. The corresponding lemma of Stewart and Leyk (Lemma 2, [12]) is similar, but derived differently, using the min-max theorem for symmetric matrices. It is

$$
\left\|e^{-\mathbf{A}} \mathbf{v}-\mathbf{Q}_{\mathrm{m}} \mathbf{e}^{-\mathbf{T}_{\mathbf{m}}} \mathbf{Q}_{\mathrm{m}}{ }^{\mathrm{T}} \mathbf{v}\right\|_{2} \leq \mathbf{2}\|\mathbf{v}\|_{2}\|\mathbf{r}\|_{1}
$$

where

$$
\|r\|=\min \max \left\{\left|e^{-\lambda}-p_{m-1}(\lambda)\right| ; \lambda \in\left[\lambda_{\min }, \lambda_{\max }\right] ;\right\}
$$

and $\lambda_{\min }$ and $\lambda_{\max }$ are the minimum and maximum eigenvalues of $\mathbf{A}$.

\subsection{Lubich Derivation}

Lubich combines his Theorem 2.9 with a bound on $\left|p_{m-1}(x)-e^{i \omega x}\right|$ (his Theorem 2.2) to derive a bound on the error. He finds that for any Hermitian matrix, $\mathbf{H}$, all of whose eigenvalues are in the interval $[a, b]$, the error of the Lanczos method is bounded by

$$
\epsilon_{b}^{H L}=|| \mathbf{Q}_{\mathbf{m}} e^{-i \Delta t \mathbf{T}_{\mathbf{m}}} \mathbf{e}_{\mathbf{1}}-e^{-i \Delta t \mathbf{H}} \mathbf{v} \| \leq 8\left(e^{1-(\omega / 2 m)^{2}} \frac{|\omega|}{2 m}\right)^{m} ; m \geq|\omega| ; \omega=\Delta t(b-a) / 2,
$$

where $\mathbf{v}$ be a vector of unit Euclidean norm. $\Delta t^{H L}$ is derived by setting $y=|\omega| / 2 m=(b-a) \Delta t / 4 m$, replacing the above inequality by an equality, and solving iteratively the following equation

$$
\epsilon_{b}^{H L}=8\left(e^{1-y^{2}} y\right)^{m}=\left\{8 e^{-m y^{2}}\right\} \alpha^{m},
$$

where $\alpha=e y$.

\subsection{Mohankumar-Carrington Derivation}

MC replace $p_{m-1(x)}$ in Equation (11) with a Chebyshev [13] approximation for $e^{-i \omega x}$ which is,

$$
J_{0}(\omega) T_{0}(x)+\sum_{n=1}^{m-1}(-i)^{n} 2 J_{n}(\omega) T_{n}(x) .
$$

In Equation (11), it is the difference of the exact function $e^{-i \omega x}$ and the polynomial (here a Chebyshev polynomial) of degree $(m-1)$ that appears. We therefore need an estimate of the remainder $R_{m}=\sum_{n=m}^{\infty}(-i)^{n} 2 J_{n}(\omega) T_{n}(x)$. Since the modulus of $T_{n}(x)$ cannot exceed unity, we get

$$
\left|R_{m}\right|<\sum_{n=m}^{\infty}\left|2 J_{n}(\omega)\right|
$$

In the Appendix A, we show that

$$
J_{n}(x)<\frac{1}{\sqrt{2 \pi n}}(e x / 2 n)^{n} .
$$

It is straightforward to obtain a closed-form expression that bounds the right hand side (RHS) of Equation (15), by using Equation (16) and summing a geometric series. This is explained in [8]. We obtain

$$
\epsilon_{b}^{M C}=\left\{\sqrt{\frac{8}{\pi m}} \frac{1}{(1-\alpha)}\right\} \alpha^{m}
$$


In both Equations (17) and (14), the dominant term is $\alpha^{m}$. In typical calculations, $\Delta t$ is small enough that $1-\alpha \sim 1$ and $e^{-m y^{2}} \sim 1$ and in this case the time step $\Delta t^{M C}$ is larger than $\Delta t^{L}$ by a factor of $(8 \pi m)^{1 / m} . \Delta t^{M C}$ is larger than $\Delta t^{L}$ whenever $\alpha<0.8$.

\section{Test Calculations for Real-Time Propagation}

To test the ideas, we propagated a Gaussian wave packet in a 1D harmonic potential. The Hamiltonian (atomic units are used) is

$$
H=\frac{p^{2}}{2 m_{0}}+\frac{1}{2} m_{0} \omega^{2} x^{2},
$$

where $m_{0}=1$ and $\omega=2.7338 \times 10^{-4}$, corresponding to a wavenumber of $60 \mathrm{~cm}^{-1}$. The starting wave packet is displaced from equilibrium by $x_{0}=56$ :

$$
\begin{aligned}
\psi(x, 0) & =\frac{\alpha_{0}^{1 / 2}}{\pi^{1 / 4}} e^{-\alpha_{0}^{2}\left(x-x_{0}\right)^{2} / 2} \\
\alpha_{0} & =\left[m_{0} k / \hbar^{2}\right]^{1 / 4} ; \omega=\left(k / m_{0}\right)^{1 / 2} .
\end{aligned}
$$

The exact solution is known [14],

$$
\|\psi(x, t)\|^{2}=\frac{\alpha_{0}}{\sqrt{\pi}} e^{-\alpha_{0}^{2}\left[x-x_{0} \cos (\omega t)\right]^{2}} .
$$

The Hamiltonian matrix was constructed using the sinc discrete variable representation (DVR) of [15]. The size of the matrix is 80 . We set $m=22$. The DVR points are between -550 and +550 bohr. The spectral range is $(b-a)=0.0309$ hartree.

Absolute values of relative errors in the propagated wave packet are shown in Table 1 . The errors are at the values of $x$ in the first column. There is one pair of columns for each $\epsilon$. The first member of a pair contains errors computed with the MC time step; the second member of a pair contains errors computed with the L time step. The MC time step is somewhat larger, but the MC and L errors are comparable. Both errors are small. The total time for which the wave packet is propagated is approximately the same for an MC and L column with the same $\epsilon$. Table 2 gives $\Delta t^{M C}, \Delta t^{L}$, and the percentage increase for several $\epsilon$ values. When $\epsilon \leq 10^{-10}, \Delta t^{M C}$ is about $10 \%$ larger.

Table 1. Results of the comparison of the time-step criteria.

\begin{tabular}{ccccccccc}
\hline Time-Step & 689.11 & 661.6 & 566.49 & 526.1 & 463.57 & 421.5 & 378.35 & 339.3 \\
Iterations & 200 & 208 & 200 & 215 & 200 & 220 & 200 & 220 \\
$\epsilon / x$ & $10^{-4}$ & $10^{-4}$ & $10^{-6}$ & $10^{-6}$ & $10^{-8}$ & $10^{-8}$ & $10^{-10}$ & $10^{-10}$ \\
\hline 12 & $1.298 \times 10^{-6}$ & $1.081 \times 10^{-6}$ & $4.925 \times 10^{-9}$ & $2.029 \times 10^{-9}$ & $2.319 \times 10^{-10}$ & $3.604 \times 10^{-11}$ & $3.287 \times 10^{-12}$ & $7.618 \times 10^{-13}$ \\
23 & $1.149 \times 10^{-7}$ & $4.520 \times 10^{-7}$ & $3.644 \times 10^{-8}$ & $6.550 \times 10^{-14}$ & $3.314 \times 10^{-10}$ & $2.538 \times 10^{-11}$ & $8.968 \times 10^{-13}$ & $1.617 \times 10^{-12}$ \\
58 & $4.238 \times 10^{-7}$ & $3.929 \times 10^{-7}$ & $6.506 \times 10^{-9}$ & $1.114 \times 10^{-9}$ & $2.821 \times 10^{-11}$ & $5.802 \times 10^{-12}$ & $4.718 \times 10^{-12}$ & $1.077 \times 10^{-12}$ \\
89 & $6.756 \times 10^{-7}$ & $1.854 \times 10^{-7}$ & $2.126 \times 10^{-11}$ & $1.396 \times 10^{-10}$ & $7.604 \times 10^{-11}$ & $2.442 \times 10^{-12}$ & $7.601 \times 10^{-12}$ & $1.121 \times 10^{-12}$ \\
120 & $9.161 \times 10^{-7}$ & $3.006 \times 10^{-7}$ & $4.231 \times 10^{-9}$ & $8.909 \times 10^{-10}$ & $1.044 \times 10^{-10}$ & $1.852 \times 10^{-11}$ & $5.667 \times 10^{-12}$ & $1.712 \times 10^{-12}$ \\
200 & $4.398 \times 10^{-7}$ & $2.536 \times 10^{-7}$ & $3.462 \times 10^{-9}$ & $3.918 \times 10^{-10}$ & $9.433 \times 10^{-11}$ & $1.276 \times 10^{-11}$ & $1.619 \times 10^{-12}$ & $1.741 \times 10^{-12}$ \\
400 & $1.931 \times 10^{-6}$ & $5.332 \times 10^{-7}$ & $3.017 \times 10^{-7}$ & $3.502 \times 10^{-8}$ & $1.824 \times 10^{-10}$ & $2.030 \times 10^{-11}$ & $9.238 \times 10^{-11}$ & $1.605 \times 10^{-13}$ \\
\hline
\end{tabular}

Table 2. Comparison of time-step sizes.

\begin{tabular}{cccc}
\hline $\boldsymbol{\epsilon}$ & $\Delta \boldsymbol{t}^{M C}$ & $\Delta \boldsymbol{t}^{L}$ & Percentage Increase \\
\hline $1 \times 10^{-4}$ & $6.891 \times 10^{2}$ & $6.616 \times 10^{2}$ & 4.15 \\
$1 \times 10^{-6}$ & $5.665 \times 10^{2}$ & $5.261 \times 10^{2}$ & 7.68 \\
$1 \times 10^{-8}$ & $4.632 \times 10^{2}$ & $4.215 \times 10^{2}$ & 9.97 \\
$1 \times 10^{-10}$ & $3.784 \times 10^{2}$ & $3.393 \times 10^{2}$ & 11.5 \\
$1 \times 10^{-12}$ & $3.083 \times 10^{2}$ & $2.739 \times 10^{2}$ & 12.6 \\
$1 \times 10^{-14}$ & $2.509 \times 10^{2}$ & $2.214 \times 10^{2}$ & 13.3 \\
\hline
\end{tabular}




\section{Imaginary Time Propagation}

It is sometimes necessary to compute $\exp (-\mathbf{A}) \mathbf{v}$, where $\mathbf{A}$ is a real matrix. This is done, for example, to compute the ground state of a quantum system [16]. Using the Chebyshev approach, we can derive an error bound for the imaginary time propagation.

\subsection{An Error Bound from a Geometric Series}

It is straightforward to derive a Chebyshev expansion of $e^{-\omega x}$, where $\omega$ is real, $\omega>0$, and $x$ is between -1 and +1 . The expansion coefficients are obtained from the integral [17]

$$
\int_{-1}^{1} \frac{e^{-\omega x} T_{n}(x) d x}{\sqrt{1-x^{2}}}=(-1)^{n} \pi I_{n}(\omega),
$$

where $I_{n}(\omega)$ is a modified Bessel function of the first kind. The expansion is

$$
e^{-\omega x}=\sum_{j=0}^{\infty} a_{j}(\omega) T_{j}(x)
$$

where

$$
a_{0}(\omega)=I_{0}(\omega) ; a_{j}=(-1)^{j} 2 I_{j}(\omega) .
$$

To use Equation (10) to bound $\left\|\mathbf{Q}_{\mathbf{m}} e^{-\Delta t \mathbf{T}_{\mathbf{m}}} \mathbf{e}_{1},-e^{-\Delta t \mathbf{H}} \mathbf{v}\right\|$, we use a Chebyshev expansion of $e^{-\Delta t z}, \Delta t>0 ; z \in[a, b]$. Both $\Delta t$ and $z$ are real. The expansion is obtained by mapping $z$ onto $x$, using $z=(a+b) / 2+x(b-a) / 2$. We find,

$$
e^{-\Delta t z}=e^{-(\Delta t / 2)(a+b)}\left\{I_{0}(\omega)+2 \sum_{j=1}^{\infty}(-1)^{j} I_{j}(\omega) T_{j}(x)\right\} ; \omega=(\Delta t / 2)(b-a) .
$$

If we truncate the series after $j=(m-1)$, then the remainder is

$$
R_{m}=2 e^{-(\Delta t / 2)(a+b)} \sum_{j=m}^{\infty}(-1)^{j} I_{j}(\omega) T_{j}(x) .
$$

Hence, the bound on $R_{m}$ is

$$
\left|R_{m}\right| \leq 2 e^{-(\Delta t / 2)(a+b)} \sum_{j=m}^{\infty}\left|I_{j}(\omega)\right| .
$$

If the argument $\omega$ of $I_{n}(\omega)$ is real, then (9.6.3, Abramowitz and Stegun, page 375 [18]),

$$
I_{j}(\omega)=e^{-i j \pi / 2} J_{j}(i \omega) .
$$

We now use the asymptotic expression for $\left|J_{n}(\omega)\right|$ (Abramowitz and Stegun, 9.3.1 [18]):

$$
\left|I_{n}(\omega)\right|=\left|e^{-i n \pi / 2} J_{n}(i \omega)\right|=\left|J_{n}(i \omega)\right| \sim \frac{1}{\sqrt{2 \pi n}}(e \omega / 2 n)^{n} .
$$

When $\omega>0$, the sum in Equation (27) is therefore bounded by a geometric series (as is true for the real-time case)

$$
\left|\sum_{j=m}^{\infty} I_{j}(\omega)\right|<\frac{1}{\sqrt{2 \pi m}} \frac{\left[\frac{e \omega}{2 m}\right]^{m}}{\left(1-\frac{e \omega}{2 m}\right)}
$$


and $\left|R_{m}\right|$ itself is bounded by

$$
\left|R_{m}\right| \leq 2 e^{-(\Delta t / 2)(a+b)} \frac{1}{\sqrt{2 \pi m}} \frac{\left[\frac{e \omega}{2 m}\right]^{m}}{\left(1-\frac{e \omega}{2 m}\right)} .
$$

The geometric series converges only if $(e \omega /(2 m))=(e \Delta t /(4 m))(b-a)<1$. Including the factor of two in Equation (10), we find that the error bound is

$$
\epsilon=4 e^{-(\Delta t / 2)(a+b)} \frac{1}{\sqrt{2 \pi m}} \frac{\left[\frac{e \omega}{2 m}\right]^{m}}{\left(1-\frac{e \omega}{2 m}\right)} .
$$

This is much simpler than the bound given by Stewart and Leyk [12].

\subsection{An Error Bound from the First Term in Equation (27)}

According to Equation (29), for a fixed $z, J_{m}(z) \sim 1 / m^{m+1 / 2}$. Hence, the first term in the series in Equation (27) is the largest. If it is significantly larger, then the first term, by itself, can be used to bound the error. If we use Equation (29) and keep only the first term, we find the error bound,

$$
E 1=\epsilon=4 e^{-(s / 2)(a+b)} I_{m}(\omega) .
$$

Note that the error bound of Equation (32) is only valid if $(e \Delta t /(4 m))(b-a)<1$, but that the error bound of Equation (33) has no such constraint.

\subsection{Test Calculations}

For the case $e^{-\mathbf{A}}$, where $\mathbf{A}$ is real and symmetric (which corresponds to imaginary time propagation), we tested Equations (32) and (33) using the $n \times n$ diagonal matrix $\mathbf{A}=\mathbf{A}_{\mathbf{2}}$, used to test the error bound of SL [12]. Its elements are $d(1)=1, d(n)=1+c, c>0$, and $d(j)=1+c x_{j}, j=$ $2,3, \ldots,(n-1), 0<x_{j}<1$, where $x_{j}$ is a uniformly distributed random number between 0 and 1 . The minimum and maximum diagonal entries are $d(1)$ and $d(n)$, which are also the minimum and

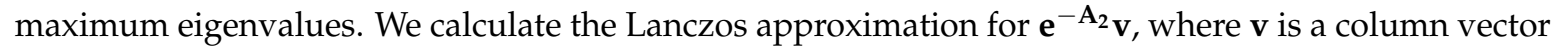
of unit norm. It is $\mathbf{Q} e^{-\Lambda} \mathbf{Q}^{\mathrm{T}} \mathbf{v}$, where $\mathbf{Q}=\mathbf{Q}_{\mathbf{m}} \mathbf{U}$ and $\mathbf{U}^{\mathrm{T}} \mathbf{T}_{\mathbf{m}} \mathbf{U}=\boldsymbol{\Lambda}$ (notation of Equation (6)). In Table 3, we give exact values of $E r r=\left|e^{-\mathbf{A}_{\mathbf{2}} \mathbf{v}}-\mathbf{Q} e^{-\Lambda} \mathbf{Q}^{\mathrm{T}} \mathbf{v}\right|$ for various values of $n, m$ and $c$, and also the errors calculated with Equation (30) and Equation (33). Note that here $\Delta t=1$. When $c$ is large, the condition $(e \Delta t / 4 \mathbf{m})(\mathbf{b}-\mathbf{a})<\mathbf{1}$ is not satisfied and we cannot sum the geometric series that leads to the error E2. The corresponding entries in the table are missing. According to Table 3, the E2 bound is conservative and the E1 bound given by Equation (33) is quite good. Both the error bounds we derive are much tighter than the error bound of Stewart and Leyk (their Equation (23)). 
Table 3. Comparison of the error bounds of Equations (33) and (32) for imaginary time propagation. $n$ is the size of the matrix; $m$ is the size of the tridiagonal matrix; $c$ is a parameter in $A_{2}$; and Err $=\left|e^{-A_{2}} v-Q e^{-\Lambda} Q^{T} v\right|$.

\begin{tabular}{ccccccc}
\hline$n$ & $m$ & $c$ & Err & Equation (33) & Equation (32) & SL Error \\
\hline 100 & 12 & 8 & $9.848 \times 10^{-8}$ & $3.125 \times 10^{-7}$ & $4.243 \times 10^{-7}$ & $\times 10^{-3}$ \\
100 & 22 & 8 & $6.149 \times 10^{-16}$ & $1.196 \times 10^{-16}$ & $1.341 \times 10^{-16}$ & $3.545 \times 10^{-3}$ \\
100 & 22 & 18 & $1.484 \times 10^{-11}$ & $9.009 \times 10^{-11}$ & $8.576 \times 10^{-11}$ & $1.437 \times 10^{-6}$ \\
200 & 12 & 6 & $7.919 \times 10^{-9}$ & $2.357 \times 10^{-8}$ & $3.027 \times 10^{-8}$ & $1.848 \times 10^{-2}$ \\
200 & 20 & 8 & $4.160 \times 10^{-15}$ & $1.404 \times 10^{-14}$ & $1.602 \times 10^{-14}$ & $3.545 \times 10^{-3}$ \\
200 & 20 & 40 & $2.594 \times 10^{-6}$ & $9.672 \times 10^{-6}$ & --- & $2.476 \times 10^{-2}$ \\
400 & 12 & 5 & $1.213 \times 10^{-9}$ & $4.136 \times 10^{-9}$ & $5.155 \times 10^{-9}$ & $4.219 \times 10^{-2}$ \\
400 & 20 & 5 & $2.685 \times 10^{-15}$ & $4.638 \times 10^{-18}$ & $5.209 \times 10^{-18}$ & $4.219 \times 10^{-2}$ \\
1200 & 100 & 20 & $1.751 \times 10^{-14}$ & $7.231 \times 10^{-93}$ & $6.541 \times 10^{-93}$ & $1.766 \times 10^{-7}$ \\
1200 & 100 & 40 & $2.842 \times 10^{-14}$ & $8.706 \times 10^{-67}$ & $4.467 \times 10^{-67}$ & $1.191 \times 10^{-14}$ \\
4000 & 12 & 15 & $1.224 \times 10^{-5}$ & $3.731 \times 10^{-5}$ & $8.790 \times 10^{-5}$ & $2.094 \times 10^{-2}$ \\
4000 & 32 & 15 & $4.078 \times 10^{-15}$ & $1.105 \times 10^{-20}$ & $1.064 \times 10^{-20}$ & $1.096 \times 10^{-5}$ \\
\hline
\end{tabular}

\section{Conclusions}

In this paper, we review and contrast two approaches [8,9] for bounding the error of a Lanczos approximation to $\exp (-i t \mathbf{H}) \mathbf{v}$, where $\mathbf{H}$ is a real matrix, $t$ is a real constant, and $\mathbf{v}$ is a real vector. In physics applications, $t$ is the time. Once an error bound has been found, it is straightforward to derive an equation for the best time step to use. The time step obtained from the MC approach [8] is slightly larger. This means that the cost of the the propagation with the MC time step is slightly less. In addition, we used the MC approach to derive an error bound (and time step) for the case when $t$ in $\exp (-i t \mathbf{H})$ is imaginary. The derivation is much simpler than the previous derivation in [12] and the error bound is much tighter.

Author Contributions: N.M. and T.C. discussed and developed the ideas. N.M. did all the numerical tests.

Funding: This research was funded by Canadian Natural Sciences and Engineering Research Council 78152.

Acknowledgments: We are grateful for the support of Canadian Natural Sciences and Engineering Research Council.

Conflicts of Interest: The authors declare no conflict of interest.

\section{Appendix A}

We use Equation (16)

$$
J_{n}(x)<\frac{1}{\sqrt{2 \pi n}}\left(\frac{x e}{2 n}\right)^{n} ; n=1,2, \ldots
$$

to derive Equation (17), the error bound for real-time propagation. Here, $n$ is a positive integer and $x$ is real positive value. This relation has not previously been proved. The standard result for the bound on the Bessel function is (Equations 9.1.62 in Ref. [18])

$$
\left|J_{v}(z)\right| \leq \frac{\left|\frac{z}{2}\right|^{v} e^{|\operatorname{Im}(z)|}}{\Gamma(v+1)} ; \quad v \geq(-1 / 2),
$$

where $z$ is complex. When $v$ is a positive integer and $z=x$ is real, Equation (A1) reduces to

$$
\left|J_{n}(x)\right| \leq \frac{\left|\frac{x}{2}\right|^{n}}{n !}
$$


An exact expression for $n$ ! is given by [19]

$$
n !=\sqrt{2 \pi} n^{n+(1 / 2)} e^{-n} e^{r_{n}} ; n=1,2, \ldots,
$$

for some $r_{n}$ in the range

$$
\frac{1}{12 p+1}<r_{p}<\frac{1}{12 p} .
$$

Since $e^{r_{p}}>1$, the two equations above imply

$$
n !>\sqrt{2 \pi} n^{n+(1 / 2)} e^{-n} ; \quad n=1,2, \ldots
$$

In view of Equations (A1) and (A5), we get for $x>0$

$$
\begin{aligned}
J_{n}(x) & \leq \frac{1}{n !}(x / 2)^{n}<\frac{(x / 2)^{n}}{\sqrt{2 \pi n} n^{n} e^{-n}} \\
& <\frac{1}{\sqrt{2 \pi n}}\left(\frac{x e}{2 n}\right)^{n} ; n=1,2, \ldots
\end{aligned}
$$

Surprisingly, the RHS is exactly equal to the usual asymptotic relation for Bessel functions (Equations 9.3.1 in Ref. [18]).

\section{References}

1. Park, T.J.; Light, J.C. Light, Unitary quantum time evolution by iterative Lanczos reduction. J. Chem. Phys. 1986, 85, 5870. [CrossRef]

2. Schinke, R. Photodissociation Dynamics; Cambridge University Press: Cambridge, UK, 1993.

3. Wyatt, R.E.; Zhang, J.Z.H. (Eds.); Dynamics of Molecules and Chemical Reaction; Marcel Dekker: New York, NY, USA, 1996.

4. Gray, S.K.; Balint-Kurti, G.G. Quantum dynamics with real wave packets, including applica- tion to three-dimensional $(\mathrm{J}=0) \mathrm{D}+\mathrm{H} 2 \rightarrow \mathrm{HD}+\mathrm{H}(\mathrm{J}=0) \mathrm{D}+\mathrm{H} 2 \rightarrow \mathrm{HD}+\mathrm{H}$ reactive scattering. J. Chem. Phys. 1998, 108, 950. [CrossRef]

5. Park, T.J.; Light, J.C. Quantum flux operators and thermal rate constant: Collinear H+H2. J. Chem. Phys. 1988, 88, 4897. [CrossRef]

6. Wei, $\mathrm{H}$.; Carrington, T. A time-dependent calculation of the alignment and orientation of the $\mathrm{CN}$ fragment of the photodissociation of ICN. J. Chem. Phys. 1996, 105, 141. [CrossRef]

7. Miller, S.M.; Carrington, T. Calculation of reaction probabilities using wavepackets. Chem. Phys. Lett. 1997, 267, 417. [CrossRef]

8. Mohankumar, N.; Carrington, T., Jr. A new approach for determining the time step when propagating with the Lanczos algorithm. Comput. Phys. Commun. 2010, 181, 1859. [CrossRef]

9. Lubich, C. From Quantum to Classical Molecular Dynamics: Reduced Models and Numerical Analysis; European Mathematical Society: Zurich, Switzerland, 2008.

10. Hochbruck, M.; Lubich, C.; Selhofer, H. Exponential integrators for large systems of differential equations. SIAM J. Sci. Comput. 1998, 19, 1552. [CrossRef]

11. Hochbruck, M.; Lubich, C. On Krylov Subspace Approximations to the Matrix Exponential Operator. SIAM J. Numer. Anal. 1997, 34, 1911. [CrossRef]

12. Stewart, D.E.; Leyk, T.S. Error estimates for Krylov subspace approximations of matrix expo-nentials. J. Comput. Appl. Math. 1996, 72, 359. [CrossRef]

13. Tal-Ezer, H.; Kosloff, R. An accurate and efficient scheme for propagating the time dependent Schrdinger equation. J. Chem. Phys. 1984, 81, 3967. [CrossRef]

14. Schiff, L.I. Quantum Mechanics, 3rd ed.; McGraw-Hill: New York, NY, USA, 1968; p. 74. 
15. Colbert, D.; Miller, W.H. A novel discrete variable representation for quantum mechanical reactive scattering via the S-matrix Kohn method. J. Chem. Phys. 1992, 96, 1982. [CrossRef]

16. Kosloff, R.; Tal-Ezer, H. A direct relaxation method for calculating eigenfunctions and eigenvalues of the schrdinger equation on a grid. Chem. Phys. Lett. 1986, 127, 223-230. [CrossRef]

17. Prudnikov, A.P.; Brychkov, Y.A.; Marichev, O.I. (Eds.) Integrals and Series; Gordon and Breach: New York, NY, USA, 1992; Volume 2, p. 453.

18. Abramowitz, M.; Stegun, I.A. Handbook of Mathematical Functions; Dover: New York, NY, USA, 1965.

19. Robbins, H. A Remark on Stirling's Formula. Am. Math. Mon. 1955, 62, 26. [CrossRef]

(C) 2019 by the authors. Licensee MDPI, Basel, Switzerland. This article is an open access article distributed under the terms and conditions of the Creative Commons Attribution (CC BY) license (http://creativecommons.org/licenses/by/4.0/). 